\title{
Review Article \\ Motor Activity in Aging: An Integrated Approach for Better Quality of Life
}

\author{
Lorenza Pratali, ${ }^{1}$ Francesca Mastorci, ${ }^{1}$ Nicola Vitiello, ${ }^{2}$ Annamaria Sironi, ${ }^{1}$ \\ Amalia Gastaldelli, ${ }^{1}$ and Angelo Gemignani ${ }^{1,3}$ \\ ${ }^{1}$ Institute of Clinical Physiology, National Research Council, Via Moruzzi 1, 56125 Pisa, Italy \\ ${ }^{2}$ The BioRobotics Institute, Scuola Superiore Sant'Anna, Polo Sant'Anna Valdera, Viale Rinaldo Piaggio 34, Pontedera, \\ 56025 Pisa, Italy \\ ${ }^{3}$ Department of Surgery, Medical, Molecular and Critical Area Pathology, University of Pisa, Via Paradisa 2, 56100 Pisa, Italy
}

Correspondence should be addressed to Lorenza Pratali; lorenza@ifc.cnr.it

Received 7 July 2014; Accepted 19 October 2014; Published 24 November 2014

Academic Editor: Maria Fiatarone Singh

Copyright (C) 2014 Lorenza Pratali et al. This is an open access article distributed under the Creative Commons Attribution License, which permits unrestricted use, distribution, and reproduction in any medium, provided the original work is properly cited.

\begin{abstract}
Old age is normally associated with stereotypical structural and physiological changes in the brain that are caused by deterioration in elementary cognitive, sensory, and sensorimotor functions as well as increased susceptibility to stress. These changes are connected with gait impairment and falls, especially among patients with common neurological diseases. Even in the absence of history of falling or when there is no physical injury after a fall, many older people develop a fear of falling that leads to restricted mobility, reduced activity, depression, social isolation, worsened metabolic disease, and increasing risk of cardiovascular morbidity and mortality. Although links between cognitive decline and age-associated brain changes have been clarified, relationships between gait disorders and psychophysiological alterations in aging are less well understood. This review focuses on two crucial elements of aged individuals with gait disorders: characteristic comorbidities in the elderly and the psychophysiological effects of physical exercise in the elderly with gait disorder. We propose an integrated approach to studying elderly subjects with gait disorder before starting a program of motor rehabilitation with wearable robotic devices, in order to investigate the effectiveness and safety of the ambulatory training.
\end{abstract}

\section{Introduction}

Our aging population is one of the most critical challenges of current industrialized societies. This issue will need to be tackled in the next few years since it threatens the sustainability of social welfare. Forty years from now, nearly $35 \%$ of the European population will be over 60 , and hence it is urgent to provide solutions enabling our aging society to remain active, creative, productive, and-above all-independent, as underlined in the "Report of Health Aging in America, 2007; Report of United Nations, 2009."

Aging is a complex phenomenon involving multiple biological pathways, from the microscopic (molecular and cellular) to macroscopic levels (tissues and organs) [1]. However, the epidemiological translation of these biological processes is quite univocal: accelerated aging diminishes healthy life expectancy. Age itself is an independent morbidity and mortality risk factor for various diseases, injuries, hospitalization, length of hospitalization, and adverse drug reactions [2,3]. Gait disorders and lower-limb impairment are common and often cause devastating quality-of-life issues for aged individuals $[4,5]$ : several population-based studies have shown a $35 \%$ prevalence of gait disorders among people over 70 years old [6] and even over 85 years old. Immobility or hypomobility due to gait disorder in aging causes decreased survival, which can be attributed to a combination of fatal falls, reduced cardiovascular fitness, and death from an underlying disease $[4,6,7]$. Usually, even in the absence of a history of falling, nearly one-third of older adults experience a fear of falling that consequently leads to self-imposed restrictions in mobility, reduced activity, depression, social isolation, and loneliness [8]. In particular, falls lead to major fractures or head trauma, and it is expected that this could occur in 500,000 individuals by the year 2040, with an expected total annual cost in 2040 of 16 billion dollars [9]. 


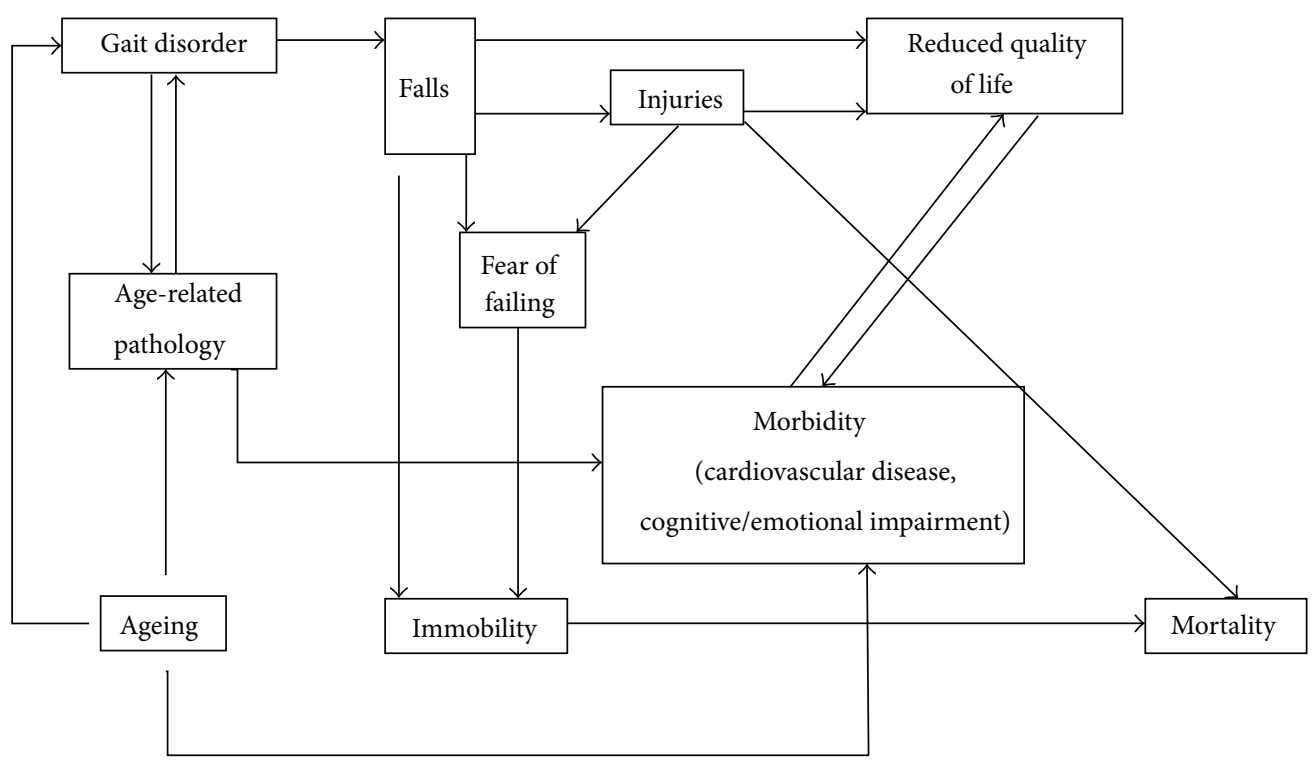

FIGURE 1: Association between aging and gait disorders. Adverse consequences of age-related gait disorders lead to reduced quality of life (depression, social isolation, and cognitive decline) and, in some cases, to mortality.

Spontaneous walking speed normally decreases by about $1 \%$ per year from the age of 60 onward [10], and the observed decline of maximum walking speed is even greater. Many older people accept their gait difficulties as normal for their age; their doctors often support them in this view.

Are Gait Disorders Truly an Inevitable Consequence of Aging Itself? Recent findings have challenged this concept: $20 \%$ of very old individuals walk normally; hence, gait disorders are certainly not an inevitable feature of old age [11]. Senile gait disorders could thus be an early manifestation of underlying pathology, most notably subtle white/grey-matter changes, vestibular, visual, or oculomotor dysfunctions. Such disorders might alter gait directly but may also act in an indirect way by causing a subjective sensation of instability and insecurity, forcing individuals to adopt a more cautious gait [12-18]. The association between aging and gait disorders is thus a complex, yet, open issue, which is well summarized by the diagram in Figure 1, adapted from Snijders et al. [4]. Aging also affects the level of cognitive involvement in gaitrelated motion tasks. Walking is traditionally seen as an automatic motor task that requires little, if any, higher mental function. However, the safety and efficacy of normal walking only on rely not sensorimotor systems, but also critically depend on the interaction between the decisions of action with the cognitive dimension. In this regard, attention is a necessary cognitive resource for maintaining normal walking and there is evidence that cognitive and attention deficits are independently associated with postural instability and future falls [19]. A common situation where such integration is challenged is when people must walk while performing a secondary task. The ability to maintain normal walking while performing a secondary task has become the classic way to assess the interaction between cognition and gait [20]. In elderly people, this ability deteriorates because central resources decline. This leads to the fact that more mental effort is required in elderly people to perform locomotion. This further increases the complexity of the task or, dually, increases the chance of gait dysfunctions. In patients with overt disease, such as stroke or Parkinson's disease, gait deteriorates even more during dual tasking [21, 22].

This review focuses on two crucial elements of aged individuals with gait disorders: characteristic comorbidities in the elderly and the effects of physical exercise in elderly persons with gait disorder. We will emphasize the presence of different comorbidities in the elderly and the importance of a multidisciplinary approach to performing studies evaluating the beneficial effect of the regular exercise in this subset of subjects. This point represents the philosophy of our perspective program of motor rehabilitation where medicine (cardiology, metabolism, and neuropsychology) meets biomedical engineering (wearable robotic devices) with the unique aim of improving the quality of life of individuals with gait disorders, in order to reduce any related medical comorbidities.

\section{Gait Disorders in the Elderly and Medical Comorbidities}

The elderly present different comorbidities in association with gait disorders, causing a stressful condition. "Stress" is the consequence of an organism becoming incapable of effectively coping with internal or environmental stimuli. Higher vulnerability and lower resilience of the stressed organism to any kind of input indicate the stress condition as the first subclinical step towards the clear manifestation of a disease, characterized by a decreased ability to maintain homeostasis [23]. In particular, chronic stress may produce cognitive dysfunction in the elderly and may increase the rate of cognitive decline in Alzheimer's patients. Stressful 
lifestyles have been suggested to increase glucocorticoid levels in the brain and this is neurotoxic, affecting neuronal energy balance, producing a decline in cognitive function, and increasing susceptibility to insomnia, depression, cardiovascular disease, and diabetes [24].

2.1. Aging and Sarcopenia. Several reports have indicated an age-related loss of skeletal muscle, that is, sarcopenia [25-28]. Elderly subjects, even when not obese (i.e., with BMI $<30 \mathrm{~kg} / \mathrm{m}^{2}$ ), have an increased percent fat mass compared to younger counterparts. The causes include medical, behavioral, and environmental factors that characterize aged individuals. The decreased muscle mass that occurs with aging has been extensively described in humans [29, 30]. It has been suggested that loss of muscle mass might be due to decreased fractional rate of muscle protein synthesis [31, 32]. However, this is not a consistently observed response. Volpi et al. $[33,34]$ have shown that under basal conditions the fractional rate of muscle protein synthesis was not impaired in apparently healthy elderly persons. Increased fasting plasma concentrations of essential amino acids (i.e., not synthesized by the human body) and in particular branched chain amino acids are often used as an indicator of protein catabolism. In the postprandial state, the availability of blood amino acids is a potent stimulus for muscle protein synthesis [35, 36] and essential amino acids are primarily responsible for this stimulatory effect [37]. In the elderly, the mechanisms associated with the stimulation of muscle protein synthesis by an elevation in blood essential amino acids are less responsive compared to younger subjects [38]. It is important to underline that sarcopenia is a reduction not only in muscle mass, but also in muscle function and can promote bone fragility due to bone mass loss and decreased bone strength. Muscle weakness, fear of falls, falls, and subsequent fractures are associated with concurrent sarcopenia and osteoporosis and lead to restricted mobility, loss of autonomy, and reduced life expectancy. The skeletal and muscular organ systems are tightly intertwined: the strongest mechanical forces applied to bones are indeed those created by muscle contractions that condition bone density, strength, and microarchitecture. For these reasons, a decrease in muscle strength is often associated with lower bone strength.

2.2. Aging and Energy Expenditure. Generally, older people tend to be increasingly sedentary; muscle disuse reduces energy requirements and appetite and the total energy expenditure (TEE) is therefore reduced. TEE is the amount of energy needed by anyone to meet their daily physical demands and has three components: (1) the amount of energy needed to maintain the body's needs at rest-basal energy expenditure-expressed as the basal metabolic rate (BMR); (2) diet-induced thermogenesis (DIT); (3) needs generated by the daily activity levels, which include physical activity grade [39]. The BMR represents the total body daily energy requirement ( $60 \%$ of TEE), which permits the function of all of the essential body systems: mainly brain, respiratory and cardiovascular function, and the work of the thermoregulatory system. Many factors can influence BMR, such as age, gender, body temperature, body weight, body composition, genetic factors, hormonal status, and pathological conditions. However, the main determinants of the BMR are fat-free mass (FFM), age, and gender [40, 41], which together account for about $80 \%$ of interindividual variability. The BMR is also associated with a decreased FFM and a lowered cellular metabolism. The FFM is the major determinant of BMR and comprises the various body organs and the skeletal muscle mass. The individual muscle mass in turn depends on age, nutrition (diet and nutritional state), and physical activity. Gender determines a lower BMR in females with respect to males with the same weight, height, and age. This difference begins at around the age of 3 and increases until puberty, when muscle mass increases in males whereas in females adipose tissue increases. BMR decreases with age, especially in the elderly, with decreased muscle mass and cellular metabolism. The DIT can be defined as the increase in energy expenditure above basal fasting level divided by the energy content of the food ingested and is commonly expressed as a percentage [42]. It accounts for $10 \%$ of TEE. DIT depends on the quality and the quantity of food introduced per meal: carbohydrates and fat have a lower thermogenesis (5$10 \%)$ with respect to protein $(20-35 \%)$. DIT is related to the stimulation of energy-requiring processes during the postprandial period. Stimulation of adenosine triphosphate hydrolysis during intestinal absorption, initial metabolic steps, and nutrient storage are responsible for this food thermic effect [43]. Physical activity is the third component of the TEE: it depends on the individual's daily activity and constitutes $15-30 \%$ of TEE. Despite the demonstrated benefits of physical activity [44], it is well known that the vast majority of older adults are physically inactive and that the prevalence of inactivity increases with advancing age. Taking into account all the TEE components, aging is associated with a TEE decline disproportionately greater than the decline in daily energy intake. Collectively, these events can create a "positive" energy balance, with secondary gains in central and total body fat, and a subsequently higher risk of morbidity and mortality [44], also accompanied by reduced muscle mass. With the growth in the number and life expectancy of older adults, the numerous risks (e.g., disability, chronic disease, reduced functional abilities, and increased falls) [45, 46], associated with the prevalence of inactivity in old age, are potentially an enormous burden not only to the older adult himself, but also to society as a whole.

2.3. Aging and Cardiovascular Disease. Aging is typically associated with cardiovascular disease, with increased evidence of systemic atherosclerosis and also a decrease in cardiovascular performance $[47,48]$. Atherosclerosis and its subsequent cardiovascular complications (myocardial infarction, stroke, and ischemic heart failure) are a major cause of death in the Western world. The risk factors of atherosclerosis are well known, including hypertension, diabetes, serum total and low-density lipoprotein cholesterol, and smoking. Increasing evidence indicates that aging is also an important risk factor for atherosclerosis and persists as an independent contributor when all other known factors are controlled. Premature or accelerated vascular aging can be promoted by cardiovascular risk factors, and cellular senescence is also 
observed in patients with atherosclerosis $[49,50]$. Atherosclerosis is therefore a disease of both organic aging and cellular senescence par excellence. Cellular senescence impairs cell proliferation, resulting in irreversible growth arrest and impaired survival, due to an accumulation of nuclear and mitochondrial DNA damage, increased reactive oxidative species, and a proinflammatory state. Both vascular aging and cellular senescence are associated with increased expression of proinflammatory cytokines and adhesion molecules further promoting inflammation and also affecting the synthesis and maintenance of extracellular matrix proteins. Aging can be identified both by structural changes and by a number of senescence-associated biomarkers. However, major gaps in our knowledge exist as to whether small changes in these biomarkers reflect an important loss of function and how aged cells promote disease. Aging is also associated with a decline in cardiovascular performance, more apparent during physical stress than at rest. The hallmarks of cardiovascular aging are reduced maximal heart rate, ejection fraction, and, in most studies, reduced maximal cardiac output with stress test $[48,51]$. The cardiovascular alterations that occur with aging in some ways parallel the changes that occur with deconditioning, including a decrease in maximal oxygen intake and maximal cardiac output. Several of the changes noted with aging are related to disuse and normalize with increased activity [52]. Although the effects of training on cardiovascular function are relatively well described in younger subjects, little is known regarding the changes that occur with training in older subjects and it has become clear that older subjects can adapt to exercise training [51, 53]. In elderly people, prevention of accelerated cell aging becomes a major therapeutic opportunity, so understanding the mechanisms contributing to changes typically associated with aging is crucial for both the prevention and development of treatment for age-related diseases.

\subsection{Aging and Cognition, Emotion, and Sleep. Aging in} humans is accompanied by stereotypical structural and physiological changes in the brain that sustain variable degrees of cognitive decline and sensorimotor dysfunction [54]. The modern neuroscientific point of view suggests that normal and especially pathological aging are not associated with cerebral regional atrophy or damage but rather with an alteration of large-scale integration between different neuronal pools, which may sustain a kaleidoscopic clinical picture, involving different aspects of brain function, from cognition to emotions, from motor to sensory, and from sleep to autonomic control [55]. Aged individuals who show delocalized activity show better cognitive performance than aged individuals with more localized activity, supporting the idea that delocalization may be a compensatory response or the effect of a residual neuronal or network plasticity [56]. These highly reliable changes occur gradually throughout middle age and are influenced by sex, age, social status, and educational background $[57,58]$. The presence of residual plasticity may constitute a barrier against age-related neurodegenerative changes [59]. In general, functional imaging studies of the human brain have revealed that cognitive functions were less coordinated, suggesting a global loss of integration, responsible for the normal activities of daily living, such as walking, dressing, or driving a vehicle [60]. This lack of coordination, typical of the elderly, will result in a higher risk of falling [61] and decreased functional and social independence [62]. In contrast with the age-related cognitive changes, emotional behavior seems to be odd, varying from an enhanced to abnormal emotional reaction such as depression $[63,64]$. Some clinical findings show that subjects experience less negative effect as they get older and can restore their good mood after a negative mood more rapidly than younger adults [65]. This peculiar emotional behavior seems to support the hypothesis that elderly have better control of their emotions, despite their cognitive decline. One possible explanation for this incongruence was provided by Cacioppo et al. [66], who showed that age-related decline in the functioning of the amygdala leads to positive effects on mood and, more generally, on emotional behavior. This amygdala-related decline selectively diminishes emotional arousal in response to negative stimuli but not to positive ones.

On the contrary, a large body of the literature indicates that aging is a vulnerable condition for developing clinical depression [67]. Furthermore, this link seems to be strongly sustained by age-related sleep changes or alterations such as insomnia [68]. Not only depression but also a large number of physical health conditions such as pain syndromes (fibromyalgia, osteoarthritis), primary pulmonary problems (chronic obstructive disease, bronchitis, and asthma), neurological disturbances (cerebrovascular accidents), and neurodegenerative disorders such as Alzheimer's disease and Parkinson's disease show a mutual relationship with sleep problems [69]. In this regard, changes in sleep architecture over the lifespan are well known [70]. From a homeostatic point of view, decreased slow-wave sleep (SWS) is a crucial factor in aging. It has been shown that the decrease in amplitude of SWS is a result of brain atrophy over time. Also, sleep spindles and K-complexes become less numerous, and the frequency of the spindles becomes slower as we age [71]. All these changes, according to Tononi and Cirelli's synaptic homeostatic hypothesis of sleep function [72], yield to a dramatic disruption of sleep-related plastic phenomena, boosting cognitive decline. It is more difficult to identify a link with depression. Adult-age depression is always characterized by decreased SWS (mainly in the first sleep cycle) [73], typically associated with an increase in hypothalamicpituitary-adrenal (HPA) axis activity or changes in neuronal plasticity (mainly in frontal areas) [74]. These two abnormal conditions are the stigmata of aging. In addition, aging is often associated with social isolation, and this condition represents a trigger factor for depression and related pathoplastic changes (decreased SWS, abnormal activation of HPA axis, and impoverishment of synaptic plasticity) [8]. Social isolation is often associated with hypomobility. It is well known that aged individuals reduce their motor activity, especially when a gait disorder is present. Motor activity is a boosting mechanism for brain plasticity, hippocampal neurogenesis, and SWS [75]. Thus, alterations in motor activity in older people can enhance their vulnerability to cognitive decline and depression. Along these lines, it has 
been shown that motor programs in older individuals with depression and insomnia improve the overall clinical picture. We also have to take into consideration that drugs used to manage chronic illnesses may also contribute to developing insomnia and depression in elderly people [76].

\section{Physical Inactivity and Aging: A Dangerous Pair}

From a physiological point of view, physical inactivity can be considered an aggression and induces the same mechanisms of stress response. This inappropriate stress response and the induced changes become risk factors for chronic disease. Immobilization is associated with decreased muscle glucose utilization (by increasing muscle insulin resistance) and triggers muscle atrophy. Both of these effects decrease further energy consumption by unused muscles. Energy is reallocated to the liver, which increases lipid production. Lipids are preferentially stored in central fat tissue. Central adipocytes are metabolically active when loaded with fat and they both produce inflammatory molecules and decrease secretion of anti-inflammatory adiponectin [77]. Moreover, the number of macrophage cells rises in fat tissue along with fat deposits. These macrophages become activated and produce proinflammatory cytokines [78, 79]. Stress response, high glucose levels, and proinflammatory cytokines increase blood coagulation and platelet aggregation and inflammation. These induced mechanisms are also critical for determining cardiovascular disease; the traditional view of atherosclerosis as a lipid storage disease crumbles in the face of extensive and growing evidence that inflammation participates centrally in all stages of this disease, from the initial lesion to the end-stage thrombotic complications [77]. Recent findings confirm that physical activity induces an increase in the systemic levels of a number of cytokines and chemokines with anti-inflammatory properties. It is possible that regular physical exercise exerts anti-inflammatory activity, since the interaction between contracting muscle and the other tissues and the circulating cells is mediated through signals transmitted by "myokines" produced with muscle contractions. To date, the list of myokines includes IL6, IL-8, and IL-15. During muscle contractions IL-1 receptor antagonist and sTNF-R, molecules that contribute to antiinflammatory actions, are also released. In spite of some discrepancies, analysis of available research seems to confirm the efficacy of regular physical training as a nonpharmacological therapy targeting chronic low-grade inflammation. Given this, physical exercise could be considered a useful weapon against local vascular and systemic inflammation in atherosclerosis. Several mechanisms explain the positive effect of chronic exercise; nevertheless, these mechanisms do not fully explain all pathways by which exercise can decrease inflammation and endothelial dysfunction and hence modulate the progression of the underlying disease progress [80]. Therefore, physical activity and aging have opposite effects on a clear end point: healthy life expectancy. Several epidemiological studies have established the benefits of physical activity for improving both life expectancy with or without disease and life expectancy in good health (healthy life expectancy) of elderly individuals. Beneficial associations between physical activity and mortality have been found in populations with various age distributions and in various geographical areas. A recent meta-analysis reviewed relevant studies of the dose-response relationship of nonvigorous physical activity and all-cause mortality [81]. These authors found that $2.5 \mathrm{~h} /$ week (equivalent to $30 \mathrm{~min}$ daily of moderate intensity activity 5 days a week) compared with no activity was associated with a reduction in mortality risk of $19 \%(95 \%$ confidence interval (CI) 15-24), while 7 h/week of moderate activity compared with no activity reduced mortality risk by 24\% (95\% CI 19-29). The largest benefit was found when moving from no activity to low levels of activity, but even at high levels of activity benefits accrue from additional activity. Moreover, recently a case-control study on the effect of the level of physical activity and the risk of myocardial infarction was published [82]. The INTERHEART study enrolled 29,000 people from 52 countries in Asia, Europe, The Middle East, Africa, Australia, and North and South America and examined how physical activity both at work and during leisure time is related with cardiovascular risk. It shows that mild to moderate physical activity at work and any level of physical activity during leisure time reduce the risk of heart attack, independent of other traditional risk factors, in men and women of all ages, in most regions of the world and in countries with low-, middle-, or high-income levels. Inactivity is a universal cardiovascular and metabolic risk factor and physical activity remains the single most neglected therapeutic intervention worldwide. The protective role of physical activity in relation to cardiovascular and metabolic disease is accompanied by an important and welldocumented involvement of motor performance in cognitive function. In particular, it is interesting to note that, among older adults, cognitive and movement disorders such as coordination difficulty [83], increased variability of movement [84], and slowing of movement [85] cooccur more frequently than what would be expected by chance, and the occurrence of either disorder raises the risk of onset of the other. Research investigating the effects of exercise on older adults has primarily focused on brain structural and functional changes with relation to cognitive improvement. In particular, several cross-sectional and intervention studies have shown a positive association between physical activity and cognition in older persons [86] and an inverse correlation with cognitive decline and dementia [87]. Older adults enrolled in a 6month aerobic fitness intervention increased brain volume in both gray matter (anterior cingulate cortex, supplementary motor area, posterior middle frontal gyrus, and left superior temporal lobe) and white matter (anterior third of corpus callosum) [88]. In addition, Colcombe and colleagues showed that older adults with higher cardiovascular fitness levels are better at activating attentional resources, including decreased activation of the anterior cingulated cortex. One of the possible mechanisms by which physical activity may benefit cognition is that physical activity maintains brain plasticity, increases brain volume, stimulates neurogenesis and synaptogenesis, and increases neurotrophic factors in different areas of the brain, possibly providing reserve against later cognitive decline and dementia $[89,90]$. 


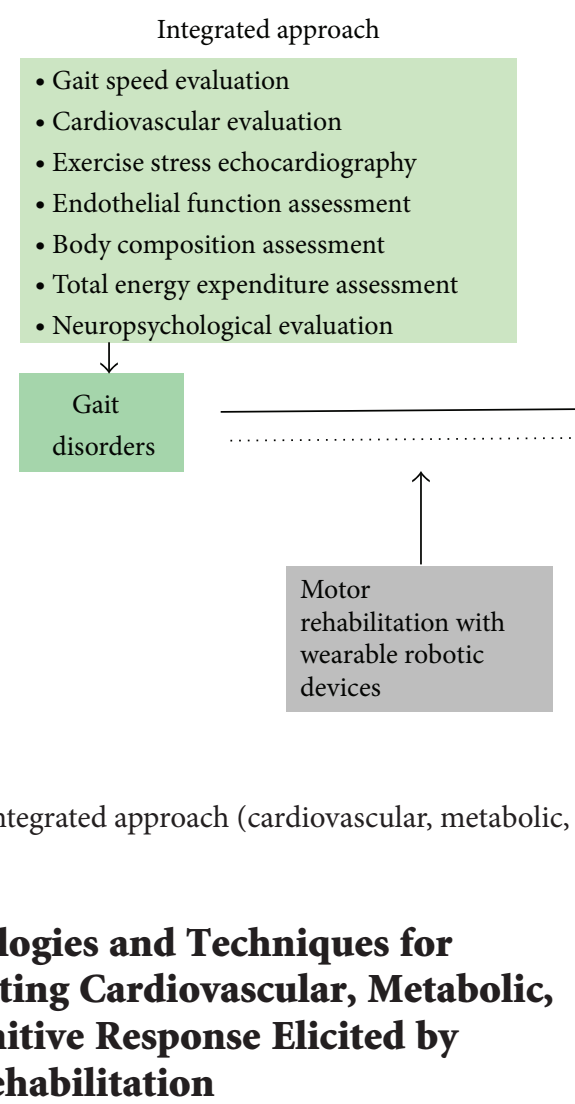

One of the main characteristics of the elderly population is its heterogeneity, and older people in the same age range show a wide variation in their risk of disability, cardiac and metabolic disease, cognitive impairment, insomnia, depression, hospitalizations, institutionalization, falls, and mortality. To prevent these adverse outcomes, population-based intervention programs should target the population at risk. Therefore, a feasible and valid screening tool available for research and clinical settings is required to identify target populations. Here we propose an integrated approach to study older subjects with gait disorder before starting a program of motor rehabilitation with wearable robotic devices to investigate the effectiveness and safety of the ambulatory training (Figure 2).

\section{Conclusion and Future Directions}

The needs of elderly people with lower-limb impairment motivated us to start a collaborative research project aimed at developing an assistive robot for the functional support of lower-limb motion. This project intends to research means of cognitive control in a multidegree-of-freedom system and to develop know-how on how humans can interface with a semiautonomous robotic device that supports a disabled person in executing locomotion-related tasks (e.g., walking, stair climbing), including transients (e.g., start, stop, sitto-stand, etc.), in a real-life unstructured environment. A multidisciplinary team (engineer, medical doctor, and therapists) will collaborate on this project to validate the new assistive robot and to investigate the acute and chronic effect of exercise in older persons with gait disorders, assisted by a wearable robot for the lower limbs.

\section{Conflict of Interests}

The authors declare that there is no conflict of interests regarding the publication of this paper.

\section{Acknowledgment}

This work was supported by IUVO Project founded by Pisa Foundation, Pisa, Italy.

\section{References}

[1] D. P. Kelly, "Cell biology: ageing theories unified," Nature, vol. 470, no. 7334, pp. 342-343, 2011.

[2] D. C. Classen, S. L. Pestotnik, R. S. Evans, J. F. Lloyd, and J. P. Burke, "Adverse drug events in hospitalized patients: excess length of stay, extra costs, and attributable mortality," The Journal of the American Medical Association, vol. 277, no. 4, pp. 301-306, 1997.

[3] J. C. Wang and M. Bennett, "Aging and atherosclerosis: mechanisms, functional consequences, and potential therapeutics for cellular senescence," Circulation Research, vol. 111, no. 2, pp. 245-259, 2012.

[4] A. H. Snijders, B. P. van de Warrenburg, N. Giladi, and B. R. Bloem, "Neurological gait disorders in elderly people: clinical approach and classification," Lancet Neurology, vol. 6, no. 1, pp. 63-74, 2007.

[5] H. Stolze, S. Klebe, C. Baecker et al., "Prevalence of Gait disorders in hospitalized neurological patients," Movement Disorders, vol. 20, no. 1, pp. 89-94, 2005.

[6] J. Verghese, A. LeValley, C. B. Hall, M. J. Katz, A. F. Ambrose, and R. B. Lipton, "Epidemiology of gait disorders in community-residing older adults," Journal of the American Geriatrics Society, vol. 54, no. 2, pp. 255-261, 2006.

[7] R. Burge, B. Dawson-Hughes, D. H. Solomon, J. B. Wong, A. King, and A. Tosteson, "Incidence and economic burden of osteoporosis-related fractures in the United States, 2005-2025," 
Journal of Bone and Mineral Research, vol. 22, no. 3, pp. 465-475, 2007.

[8] N. Valtorta and B. Hanratty, "Loneliness, isolation and the health of older adults: do we need a new research agenda?" Journal of the Royal Society of Medicine, vol. 105, no. 12, pp. 518522, 2012.

[9] R. S. Wilson, J. A. Schneider, L. A. Beckett, D. A. Evans, and D. A. Bennett, "Progression of gait disorder and rigidity and risk of death in older persons," Neurology, vol. 58, no. 12, pp. 1815-1819, 2002.

[10] J. A. Ashton-Miller, "Age-associated changes in the biomechanics of gait and gait-related falls in older adults," in Gait Disorders: Evaluation and Management, J. M. Hausdorff and N. B. Alexander, Eds., Taylor \& Francis, Boca Raton, Fla, USA, 2005.

[11] B. R. Bloem, J. Haan, A. M. Lagaay, W. van Beek, A. R. Wintzen, and R. A. C. Roos, "Investigation of gait in elderly subjects over 88 years of age," Journal of Geriatric Psychiatry and Neurology, vol. 5, no. 2, pp. 78-84, 1992.

[12] R. W. Baloh, S. H. Ying, and K. M. Jacobson, "A longitudinal study of gait and balance dysfunction in normal older people," Archives of Neurology, vol. 60, no. 6, pp. 835-839, 2003.

[13] R. R. Benson, C. R. G. Guttmann, X. Wei et al., "Older people with impaired mobility have specific loci of periventricular abnormality on MRI," Neurology, vol. 58, no. 1, pp. 48-55, 2002.

[14] T. D. Fife and R. W. Baloh, "Disequilibrium of unknown cause in older people," Annals of Neurology, vol. 34, no. 5, pp. 694-702, 1993.

[15] K. A. Kerber, G. P. Ishiyama, and R. W. Baloh, "A longitudinal study of oculomotor function in normal older people," Neurobiology of Aging, vol. 27, no. 9, pp. 1346-1353, 2006.

[16] A. Kesler, G. Leibovich, T. Herman, L. Gruendlinger, N. Giladi, and J. M. Hausdorff, "Shedding light on walking in the dark: the effects of reduced lighting on the gait of older adults with a higher-level gait disorder and controls," Journal of NeuroEngineering and Rehabilitation, vol. 2, article 27, 2005.

[17] G. T. Whitman, T. Tang, A. Lin, and R. W. Baloh, "A prospective study of cerebral white matter abnormalities in older people with gait dysfunction," Neurology, vol. 57, no. 6, pp. 990-994, 2001.

[18] L. Wolfson, X. Wei, C. B. Hall et al., "Accrual of MRI white matter abnormalities in elderly with normal and impaired mobility," Journal of the Neurological Sciences, vol. 232, no. 1-2, pp. 23-27, 2005.

[19] M. Woollacott and A. Shumway-Cook, "Attention and the control of posture and gait: a review of an emerging area of research," Gait and Posture, vol. 16, no. 1, pp. 1-14, 2002.

[20] L. Lundin-Olsson, L. Nyberg, and Y. Gustafson, "Stops walking when talking' as a predictor of falls in elderly people," The Lancet, vol. 349, no. 9052, p. 617, 1997.

[21] B. R. Bloem, Y. A. Grimbergen, J. G. van Dijk, and M. Munneke, "The "posture second" strategy: a review of wrong priorities in Parkinson's disease," Journal of the Neurological Sciences, vol. 248, no. 1-2, pp. 196-204, 2006.

[22] B. R. Bloem, V. V. Valkenburg, M. Slabbekoorn, and M. D. Willemsen, "The Multiple Tasks Test: development and normal strategies," Gait and Posture, vol. 14, no. 3, pp. 191-202, 2001.

[23] P. Sterling and J. Eyer, "Allostasis: a new paradigm to explain arousal pathology," in Handbook of Life Stress, Cognition and Health, S. Fisher and J. Reason, Eds., pp. 629-649, John Wiley \& Sons, New York, NY, USA, 1988.
[24] B. S. McEwen, "Sex, stress and the hippocampus: allostasis, allostatic load and the aging process," Neurobiology of Aging, vol. 23, no. 5, pp. 921-939, 2002.

[25] S. H. Cohn, D. Vartsky, S. Yasumura et al., "Compartmental body composition based on total-body nitrogen, potassium, and calcium," The American Journal of Physiology, vol. 239, no. 6, pp. E524-E530, 1980.

[26] C. Dutta and E. C. Hadley, "The significance of sarcopenia in old age," The Journals of Gerontology Series A Biological Sciences and Medical Sciences, vol. 50, pp. 1-4, 1995.

[27] G. B. Forbes and J. C. Reina, "Adult lean body mass declines with age: some longitudinal observations," Metabolism, vol. 19, no. 9, pp. 653-663, 1970.

[28] J. J. Kehayias, M. A. Fiatarone, H. Zhuang, and R. Roubenoff, "Total body potassium and body fat: relevance to aging," The American Journal of Clinical Nutrition, vol. 66, no. 4, pp. 904910, 1997.

[29] W. J. Evans, "What is sarcopenia?" The Journals of Gerontology, vol. 50, pp. 5-8, 1995.

[30] G. B. Forbes, "The adult decline in lean body mass," Human Biology, vol. 48, no. 1, pp. 161-173, 1976.

[31] P. Balagopal, O. E. Rooyackers, D. B. Adey, P. A. Ades, and K. S. Nair, "Effects of aging on in vivo synthesis of skeletal muscle myosin heavy-chain and sarcoplasmic protein in humans," American Journal of Physiology: Endocrinology and Metabolism, vol. 273, no. 4, pp. E790-E800, 1997.

[32] S. Welle, C. Thornton, R. Jozefowicz, and M. Statt, "Myofibrillar protein synthesis in young and old men," The American Journal of Physiology-Endocrinology and Metabolism, vol. 264, no. 5, pp. 693-698, 1993.

[33] E. Volpi, B. Mittendorfer, S. E. Wolf, and R. R. Wolfe, "Oral amino acids stimulate muscle protein anabolism in the elderly despite higher first-pass splanchnic extraction," American Journal of Physiology-Endocrinology and Metabolism, vol. 277, no. 3, pp. E513-E520, 1999.

[34] E. Volpi, M. Sheffield-Moore, B. B. Rasmussen, and R. R. Wolfe, "Basal muscle amino acid kinetics and protein synthesis in healthy young and older men," The Journal of the American Medical Association, vol. 286, no. 10, pp. 1206-1212, 2001.

[35] M. J. Rennie, J. Bohé, and R. R. Wolfe, "Latency, duration and dose response relationships of amino acid effects on human muscle protein synthesis," Journal of Nutrition, vol. 132, no. 10, pp. 3225-3227, 2002.

[36] R. R. Wolfe, "Regulation of muscle protein by amino acids," Journal of Nutrition, vol. 132, no. 10, pp. 3219-3224, 2002.

[37] E. Volpi, H. Kobayashi, M. Sheffield-Moore, B. Mittendorfer, and R. R. Wolfe, "Essential amino acids are primarily responsible for the amino acid stimulation of muscle protein anabolism in healthy elderly adults," The American Journal of Clinical Nutrition, vol. 78, no. 2, pp. 250-258, 2003.

[38] C. S. Katsanos, H. Kobayashi, M. Sheffield-Moore, A. Aarsland, and R. R. Wolfe, "Aging is associated with diminished accretion of muscle proteins after the ingestion of a small bolus of essential amino acids," The American Journal of Clinical Nutrition, vol. 82, no. 5, pp. 1065-1073, 2005.

[39] W. T. Donahoo, J. A. Levine, and E. L. Melanson, "Variability in energy expenditure and its components," Current Opinion in Clinical Nutrition \& Metabolic Care, vol. 7, no. 6, pp. 599-605, 2004.

[40] A. M. Johnstone, S. D. Murison, J. S. Duncan, K. A. Rance, and J. R. Speakman, "Factors influencing variation in basal metabolic 
rate include fat-free mass, fat mass, age, and circulating thyroxine but not sex, circulating leptin, or triiodothyronine," The American Journal of Clinical Nutrition, vol. 82, no. 5, pp. 941948, 2005.

[41] S. Lazzer, G. Bedogni, C. L. Lafortuna et al., "Relationship between basal metabolic rate, gender, age, and body composition in 8,780 white obese subjects," Obesity, vol. 18, no. 1, pp. 71-78, 2010.

[42] R. D. Starling, "Energy expenditure and aging: effects of physical activity," International Journal of Sport Nutrition and Exercise Metabolism, vol. 11, pp. S208-S217, 2001.

[43] L. Tappy, "Thermic effect of food and sympathetic nervous system activity in humans," Reproduction Nutrition Development, vol. 36, no. 4, pp. 391-397, 1996.

[44] M. E. Nelson, W. J. Rejeski, S. N. Blair et al., "Physical activity and public health in older adults: recommendation from the American College of Sports Medicine and the American Heart Association," Circulation, vol. 116, no. 9, pp. 1094-1105, 2007.

[45] W. J. Chodzko-Zajko, D. N. Proctor, M. A. F. Singh et al., "Exercise and physical activity for older adults," Medicine \& Science in Sports \& Exercise, vol. 41, no. 7, pp. 1510-1530, 2009.

[46] D. E. R. Warburton, C. W. Nicol, and S. S. D. Bredin, "Health benefits of physical activity: the evidence," Canadian Medical Association Journal, vol. 174, no. 6, pp. 801-809, 2006.

[47] E. Bolton and C. Rajkumar, "The ageing cardiovascular system," Reviews in Clinical Gerontology, vol. 21, no. 2, pp. 99-109, 2011.

[48] S. Port, F. R. Cobb, R. E. Coleman, and R. H. Jones, "Effect of age on the response of the left ventricular ejection fraction to exercise," The New England Journal of Medicine, vol. 303, no. 20, pp. 1133-1137, 1980.

[49] N. Farhat, N. Thorin-Trescases, G. Voghel et al., "Stressinduced senescence predominates in endothelial cells isolated from atherosclerotic chronic smokers," Canadian Journal of Physiology and Pharmacology, vol. 86, no. 11, pp. 761-769, 2008.

[50] B. Niemann, Y. Chen, M. Teschner, L. Li, R.-E. Silber, and S. Rohrbach, "Obesity induces signs of premature cardiac aging in younger patients: the role of mitochondria," Journal of the American College of Cardiology, vol. 57, no. 5, pp. 577-585, 2011.

[51] J. R. Stratton, W. C. Levy, M. D. Cerqueira, R. S. Schwartz, and I. B. Abrass, "Cardiovascular responses to exercise: effects of aging and exercise training in healthy men," Circulation, vol. 89 , no. 4 , pp. 1648-1655, 1994.

[52] W. M. Bortz, "Disuse and aging," The Journal of the American Medical Association, vol. 248, no. 10, pp. 1203-1208, 1982.

[53] A. A. Ehsani, T. Ogawa, T. R. Miller, R. J. Spina, and S. M. Jilka, "Exercise training improves left ventricular systolic function in older men," Circulation, vol. 83, no. 1, pp. 96-103, 1991.

[54] T. Hedden and J. D. E. Gabrieli, "Insights into the ageing mind: a view from cognitive neuroscience," Nature Reviews Neuroscience, vol. 5, no. 2, pp. 87-96, 2004.

[55] N. A. Bishop, T. Lu, and B. A. Yankner, "Neural mechanisms of ageing and cognitive decline," Nature, vol. 464, no. 7288, pp. 529-535, 2010.

[56] R. Cabeza, N. D. Anderson, J. K. Locantore, and A. R. McIntosh, "Aging gracefully: compensatory brain activity in highperforming older adults," NeuroImage, vol. 17, no. 3, pp. 13941402, 2002.

[57] H. L. Park, J. E. O’Connell, and R. G. Thomson, "A systematic review of cognitive decline in the general elderly population," International Journal of Geriatric Psychiatry, vol. 18, no. 12, pp. 1121-1134, 2003.
[58] P. A. Reuter-Lorenz, "New visions of the aging mind and brain," Trends in Cognitive Sciences, vol. 6, no. 9, pp. 394-400, 2002.

[59] R. L. Buckner, "Memory and executive function in aging and ad: Multiple factors that cause decline and reserve factors that compensate," Neuron, vol. 44, no. 1, pp. 195-208, 2004.

[60] J. R. Andrews-Hanna, A. Z. Snyder, J. L. Vincent et al., "Disruption of large-scale brain systems in advanced aging," Neuron, vol. 56, no. 5, pp. 924-935, 2007.

[61] N. de Rekeneire, M. Visser, R. Peila et al., "Is a fall just a fall: correlates of falling in healthy older persons. The health, aging and body composition study," Journal of the American Geriatrics Society, vol. 51, no. 6, pp. 841-846, 2003.

[62] W. W. Spirduso, "Issues of quantity and quality of life," in Physical Dimensions of Aging, pp. 5-30, Human Kinetics, Champaign, Ill, USA, 1995.

[63] M. Mather, "The emotion paradox in the aging brain," Annals of the New York Academy of Sciences, vol. 1251, no. 1, pp. 33-49, 2012.

[64] K. Nashiro, M. Sakaki, and M. Mather, "Age differences in brain activity during emotion processing: reflections of age-related decline or increased emotion regulation?" Gerontology, vol. 58, no. 2, pp. 156-163, 2012.

[65] N. Kryla-Lighthall and M. Mather, "The role of cognitive control in older adults' emotional well-being," in Handbook of Theories of Aging, V. Berngtson, D. Gans, N. Putney, and M. Silverstein, Eds., pp. 323-344, Springer, New York, NY, USA, 2nd edition, 2009.

[66] J. T. Cacioppo, G. G. Berntson, A. Bechara, D. Tranel, and L. C. Hawkley, "Could an aging brain contribute to subjective wellbeing? The value added by a social neuroscience perspective," in Social Neuroscience: Toward Understanding the Underpinnings of the Social Mind, A. Todorov, S. Fiske, and D. Prentice, Eds., p. 249, Oxford University Press, New York, NY, USA, 2011.

[67] E. Sibille, "Molecular aging of the brain, neuroplasticity, and vulnerability to depression and other brain-related disorders," Dialogues in Clinical Neuroscience, vol. 15, no. 1, pp. 53-65, 2013.

[68] I. Campos Costa, H. Nogueira Carvalho, and L. Fernandes, "Aging, circadian rhythms and depressive disorders: a review," The American Journal of Neurodegenerative Disease, vol. 2, no. 4, pp. 228-224, 2013.

[69] A. Y. Avidan, "Sleep changes and disorders in the elderly patient," Current Neurology and Neuroscience Reports, vol. 2, no. 2, pp. 178-185, 2002.

[70] M. M. Ohayon, M. A. Carskadon, C. Guilleminault, and M. V. Vitiello, "Meta-analysis of quantitative sleep parameters from childhood to old age in healthy individuals: developing normative sleep values across the human lifespan," Sleep, vol. 27, no. 7, pp. 1255-1273, 2004.

[71] A. Wauquier, "Aging and changes in phasic events during sleep," Physiology and Behavior, vol. 54, no. 4, pp. 803-806, 1993.

[72] G. Tononi and C. Cirelli, "Sleep and synaptic homeostasis: a hypothesis," Brain Research Bulletin, vol. 62, no. 2, pp. 143-150, 2003.

[73] M. E. Thase, "Depression and sleep: pathophysiology and treatment," Dialogues in Clinical Neuroscience, vol. 8, no. 2, pp. 217226, 2006.

[74] S. J. Lupien, B. S. McEwen, M. R. Gunnar, and C. Heim, "Effects of stress throughout the lifespan on the brain, behaviour and cognition," Nature Reviews Neuroscience, vol. 10, no. 6, pp. 434$445,2009$. 
[75] P. J. Lucassen, P. Meerlo, A. S. Naylor et al., "Regulation of adult neurogenesis by stress, sleep disruption, exercise and inflammation: implications for depression and antidepressant action," European Neuropsychopharmacology, vol. 20, no. 1, pp. $1-17,2010$.

[76] S. Ancoli-Israel, "Insomnia in the elderly: a review for the primary care practitioner," Sleep, vol. 23, no. 1, pp. 23-30, 2000.

[77] M. Morelli, M. Gaggini, G. G. Daniele, P. Marraccini, R. Sicari, and A. Gastaldelli, "Ectopic fat: the true culprit linking obesity and cardiovascular disease?" Thrombosis and Haemostasis, vol. 110, no. 4, pp. 651-660, 2013.

[78] D. Heber, "An integrative view of obesity," The American Journal of Clinical Nutrition, vol. 91, no. 1, pp. 280-283, 2010.

[79] R. M. Maizels and J. E. Allen, "Eosinophils forestall obesity," Science, vol. 332, no. 6026, pp. 186-187, 2011.

[80] P. M. Ridker, C. H. Hennekens, J. E. Buring, and N. Rifai, "C-reactive protein and other markers of inflammation in the prediction of cardiovascular disease in women," The New England Journal of Medicine, vol. 342, no. 12, pp. 836-843, 2000.

[81] J. Woodcock, O. H. Franco, N. Orsini, and I. Roberts, "Nonvigorous physical activity and all-cause mortality: systematic review and meta-analysis of cohort studies," International Journal of Epidemiology, vol. 40, no. 1, Article ID dyq104, pp. 121-138, 2011.

[82] C. Held, R. Iqbal, S. A. Lear et al., "Physical activity levels, ownership of goods promoting sedentary behaviour and risk of myocardial infarction: results of the INTERHEART study," European Heart Journal, vol. 33, no. 4, pp. 452-466, 2012.

[83] R. D. Seidler, J. L. Alberts, and G. E. Stelmach, "Changes in multi-joint performance with age.," Motor control, vol. 6, no. 1, pp. 19-31, 2002.

[84] J. L. Contreras-Vidal, H. L. Teulings, and G. E. Stelmach, "Elderly subjects are impaired in spatial coordination in fine motor control," Acta Psychologica, vol. 100, no. 1-2, pp. 25-35, 1998.

[85] V. Diggles-Buckles, "Age-related slowing," in Sensorimotor Impairments in the Elderly, G. E. Stelmach and V. Homberg, Eds., pp. 73-88, Kluwer Academic Publishers, Dordrecht, The Netherlands, 1993.

[86] W. J. Chodzko-Zajko and K. A. Moore, "Physical fitness and cognitive functioning in aging.," Exercise and Sport Sciences Reviews, vol. 22, pp. 195-220, 1994.

[87] D. Laurin, R. Verreault, J. Lindsay, K. MacPherson, and K. Rockwood, "Physical activity and risk of cognitive impairment and dementia in elderly persons," Archives of Neurology, vol. 58, no. 3, pp. 498-504, 2001.

[88] S. J. Colcombe, K. I. Erickson, P. E. Scalf et al., "Aerobic exercise training increases brain volume in aging humans," The Journals of Gerontology, vol. 61, no. 11, pp. 1166-1170, 2006.

[89] C. H. Hillman, K. I. Erickson, and A. F. Kramer, "Be smart, exercise your heart: exercise effects on brain and cognition," Nature Reviews Neuroscience, vol. 9, no. 1, pp. 58-65, 2008.

[90] F. Mora, "Successful brain aging: lasticity, environmental enrichment, and lifestyle," Dialogues in Clinical Neuroscience, vol. 15, no. 1, pp. 45-52, 2013. 


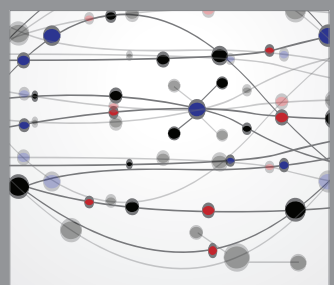

The Scientific World Journal
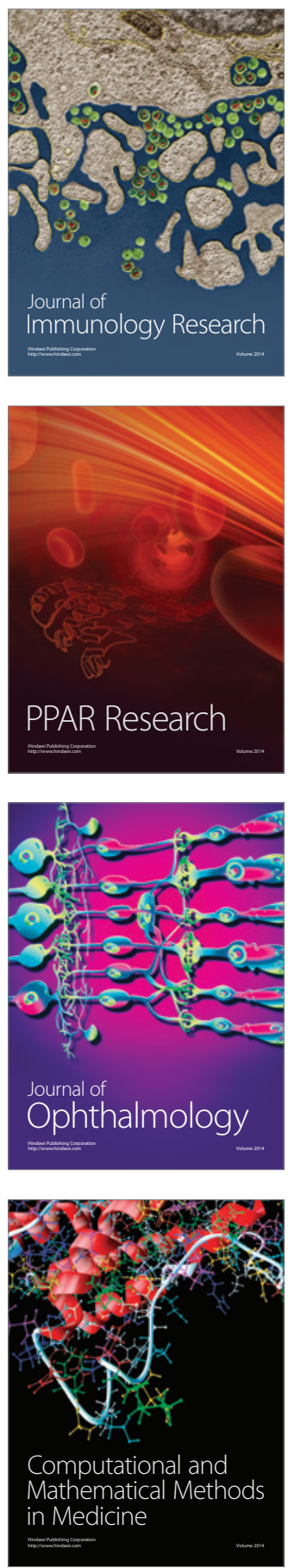

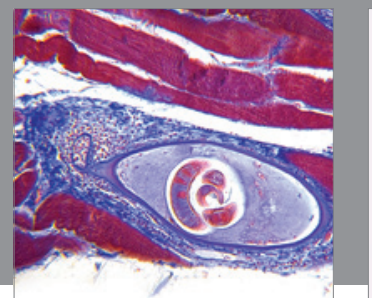

Gastroenterology

Research and Practice
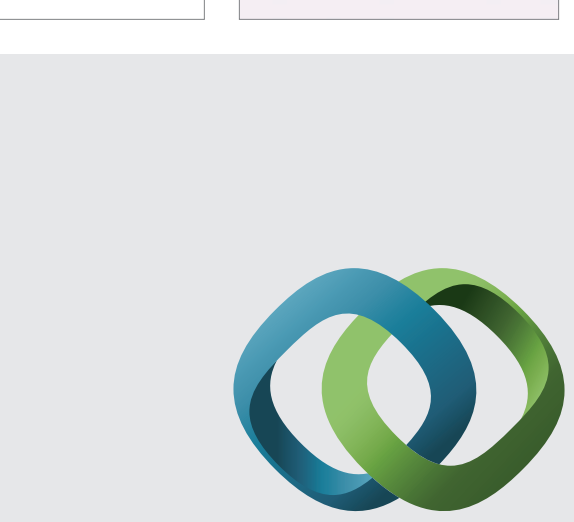

\section{Hindawi}

Submit your manuscripts at

http://www.hindawi.com
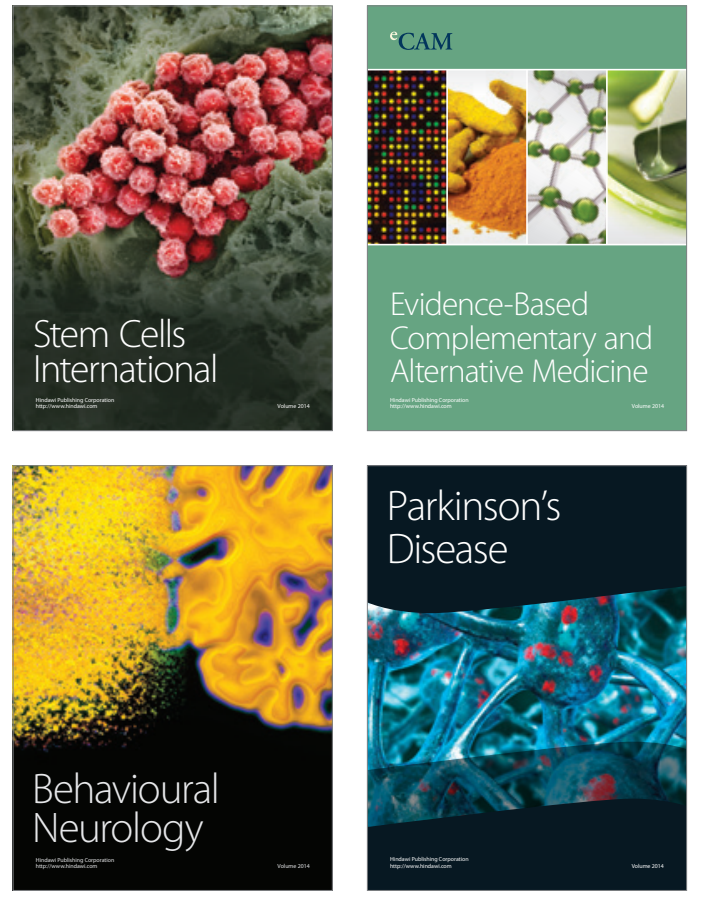
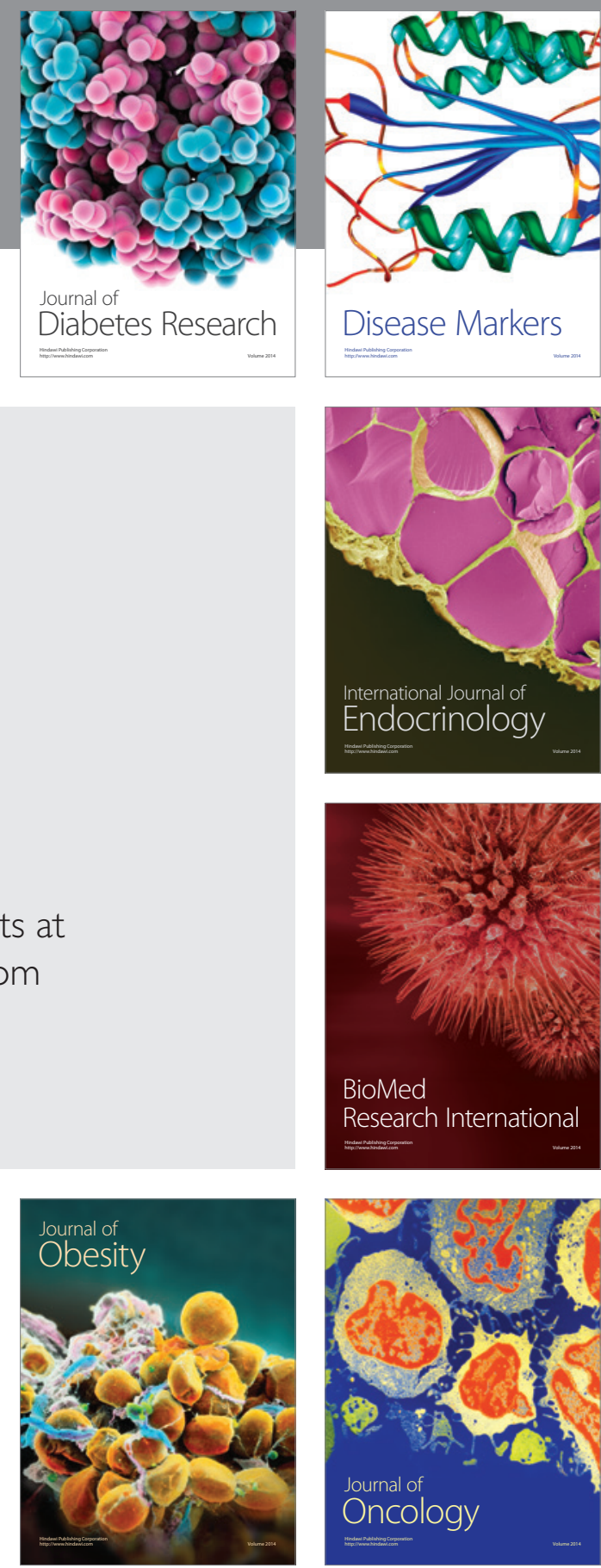

Disease Markers
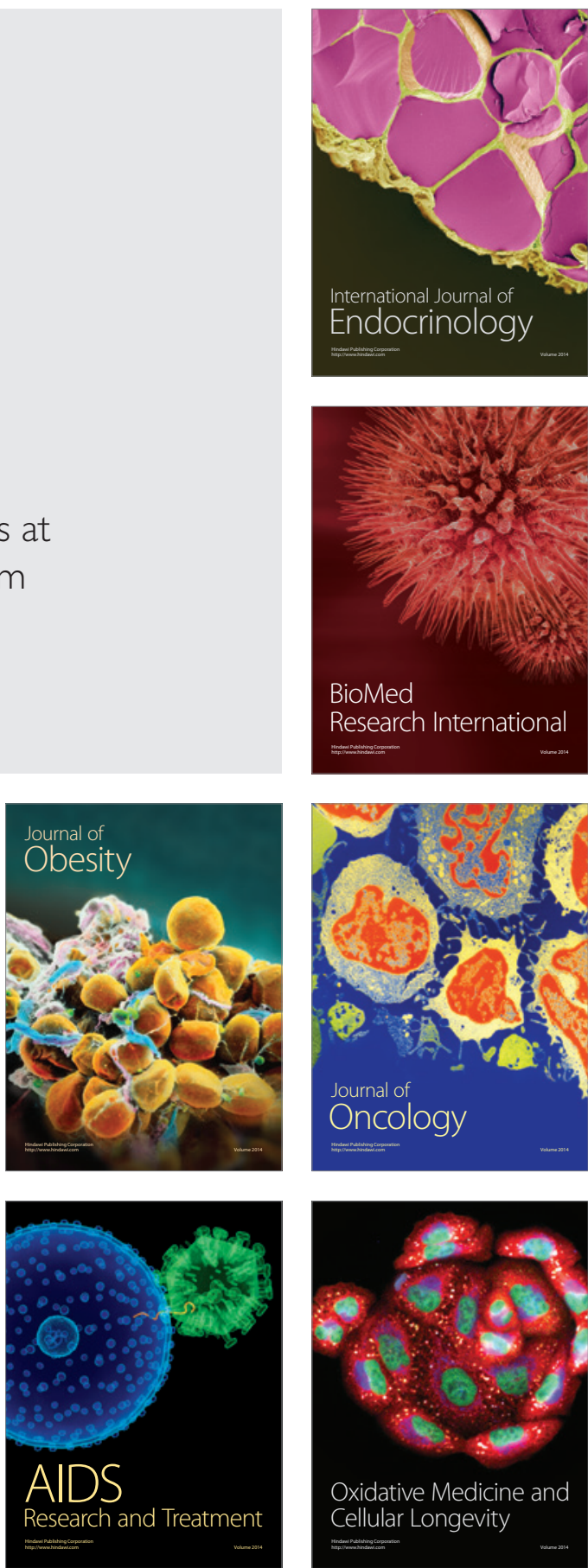